\title{
Herwig 7.2 release note
}

\author{
Johannes Bellm ${ }^{1}$, Gavin Bewick ${ }^{2}$, Silvia Ferrario Ravasio $^{2}$, Stefan Gieseke ${ }^{3}$, David Grellscheid $^{4}$, \\ Patrick Kirchgaeßer ${ }^{3}$, Frashër Loshaj ${ }^{3}$, Mohammad R. Masouminia ${ }^{2}$, Graeme Nail ${ }^{5}$, Andreas Papaefstathiou ${ }^{5}$, \\ Simon Plätzer ${ }^{6}$, Radek Podskubka $^{3}$, Michael Rauch ${ }^{3}$, Christian Reuschle $^{1}$, Peter Richardson ${ }^{2,7}$, Peter Schichtel $^{2}$, \\ Michael H. Seymour ${ }^{8, a}{ }^{\infty}$, Andrzej Siódmok ${ }^{9,10}$, Stephen Webster ${ }^{2}$ \\ ${ }^{1}$ Department of Astronomy and Theoretical Physics, Lund University, Lund, Sweden \\ 2 IPPP, Department of Physics, Durham University, Durham, UK \\ ${ }^{3}$ Institute for Theoretical Physics, Karlsruhe Institute of Technology, Karlsruhe, Germany \\ ${ }^{4}$ Department of Informatics, University of Bergen, Bergen, Norway \\ ${ }^{5}$ Higgs Centre for Theoretical Physics, University of Edinburgh, Edinburgh, UK \\ ${ }^{6}$ Particle Physics, Faculty of Physics, University of Vienna, Vienna, Austria \\ ${ }^{7}$ CERN, PH-TH, Geneva, Switzerland \\ ${ }^{8}$ Particle Physics Group, Department of Physics and Astronomy, University of Manchester, Manchester, UK \\ ${ }^{9}$ The Henryk Niewodniczański Institute of Nuclear Physics in Cracow, Polish Academy of Sciences, Warsaw, Poland \\ ${ }^{10}$ Czech Technical University in Prague, Brehova 7, 11519 Prague, Czech Republic
}

\begin{abstract}
A new release of the Monte Carlo event generator Herwig (version 7.2) is now available. This version introduces a number of improvements over the major version 7.0, notably: multi-jet merging with the dipole shower at LO and NLO QCD; spin correlations in both the dipole and angular-ordered parton showers; an improved choice of evolution variable in the angular-ordered parton shower; improvements to mass effects and top decays in the dipole shower, improvements to the simulation of multiple-parton interactions, including diffractive processes; a new model for baryonic colour reconnection; improvements to strangeness production; as well as a new tune of the hadronisation parameters and support for generic Lorentz structures in BSM models. This article illustrates new features of versions 7.1 and 7.2 .
\end{abstract}

\section{Introduction}

Herwig is a multi purpose particle physics event generator. The current version series, Herwig7 [1], is based on a major development of the Herwig++ [2-7] branch. It fully supersedes the Herwig++ 2.x and HERWIG 6.x versions. Building on the technology and experience gained with the higherorder improvements provided by Herwig 7.0 [1] and 7.1 [8], a major follow-up release, Herwig 7.2 is now available. This release note briefly summarises the main physics and techni-

\footnotetext{
a e-mail: michael.seymour@manchester.ac.uk (corresponding author)
}

cal improvements made since the last published release note (7.0 [1]). Version 7.1 provided multijet merging at next-toleading order QCD [9] as one of its main new features, as well as several improvements to the soft components of the simulation. Version 7.2 includes several further improvements to the soft components, amongst other changes and physics capabilities. These and further more minor improvements are discussed below.

Please refer to the Herwig++ manual [2], the Herwig 7.0 [1] as well as this release note when using the new version of the program. Studies or analyses that rely on a particular feature of the program should also reference the paper(s) where the physics of that feature was first described. The authors are happy to provide guidance on which features are relevant for a particular analysis.

\subsection{Availability}

The new version, as well as older versions of the Herwig event generator can be downloaded from the website https://herwig.hepforge.org/. We strongly recommend using the bootstrap script provided for the convenient installation of Herwig and all of its dependencies, which can be obtained from the same location. On the website, comparisons of Herwig 7 with large amount of experimental data, tutorials and FAQ sections are provided to help with the usage of the program. Further enquiries should be directed to herwig@projects . hepforge. org. Herwig is released under the GNU General Public License (GPL) 
version 3 and the MCnet guidelines for the distribution and usage of event generator software in an academic setting, see the source code archive or http://www.montecarlonet.org/.

\subsection{Prerequisites and further details}

Herwig 7.2 is built on the same backbone and dependencies as its predecessors Herwig 7.0 and 7.1, and uses the same method of build, installation and run environment. No major changes should hence be required in comparison to a working Herwig 7.1 installation. Some of the changes, though, might require different compiler versions. The tutorials at https://herwig.hepforge.org/tutorials/ have been extended and adapted to the new version and serve as the primary reference for physics setups and as a user manual until a comprehensive replacement for the detailed manual [2] is available.

\section{Merging and matching}

\subsection{Multijet merging}

Based on the Matchbox development [10] which is central to the NLO matching capabilities of Herwig, a multijet merging algorithm detailed in [9] has been implemented together with the dipole shower algorithm and based on an improved, unitarised merging prescription following the proposal set out in [11]. The algorithm is able to merge cross sections for multiple jet production at the NLO QCD level, and has been tested with a range of standard model processes such as vector boson or Higgs boson plus jets production, top pair production, and pure jet production.

Compared to the simple input file structure of the Matchbox framework, minor additional commands are needed to perform calculations with several jet multiplicities merged to the dipole shower. Input file examples for a range of processes are provided in share/Herwig/Merging. Different from the standard NLO matching input files for use with Matchbox, merging only requires a slightly different process definition. For example,

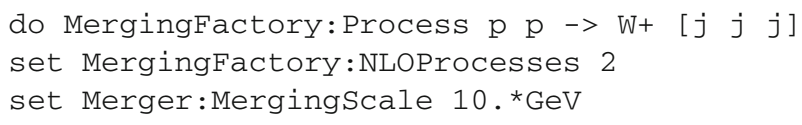

sets up on-shell $W^{+}$production with up to three jets and including NLO QCD corrections to the inclusive and one-jet process. For the merging scale we recommend some default ranges (LHC at $13 \mathrm{TeV}$ : $10-30 \mathrm{GeV}$, LEP at $91 \mathrm{GeV}$ : 4-6 $\mathrm{GeV}$ and for HERA run 2 with $27 \mathrm{GeV}$ electrons/positrons on $820 \mathrm{GeV}$ protons we have found that a merging scale between 8 and $15 \mathrm{GeV}$ has provided reliable results). For colliders running significantly outside these parameters, and
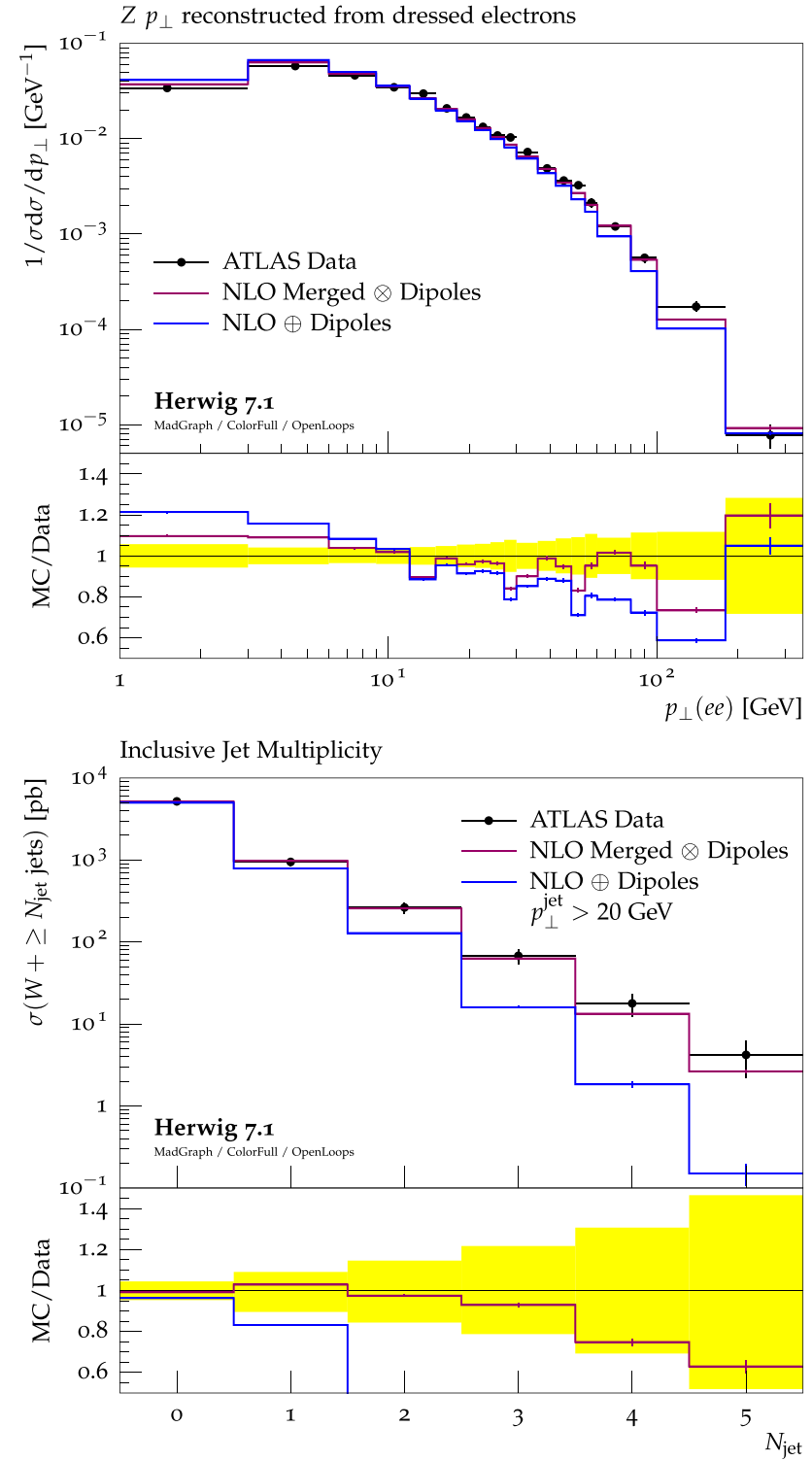

Fig. 1 The normalized $Z p_{\perp}$ spectrum (top panel), and jet multiplicites in $W$ plus jets events (lower panel) as measured by ATLAS [12,13] and comparing the NLO matched prediction with the dipole shower to the NLO multijet merged prediction. Higher jet multiplicities and less inclusive quantities will receive bigger corrections through the merging algorithm. For these results we have used our run-time interfaces to MadGraph5_aMCatNLO [14] and OpenLoops [15] to evaluate scattering amplitudes for each phase space point, and ColorFull [16] to perform the colour algebra

in dependence on acceptance cuts, the value needs to be adjusted, possibly down to small merging scales. This provides stable predictions due to the unitarisation procedure.

Example plots are shown in Fig. 1, highlighting the fact that inclusive quantities do not receive big corrections, while higher jet multiplicities are significantly improved by the procedure. Variations of the factorization and renormalization scales can be obtained as with all other simulation setups. 


\subsection{KrkNLO}

This version of Herwig contains an implementation of the KrkNLO method [17]. This provides NLO QCD corrections to LO matrix elements for specific processes following this paradigm as an alternative to the other matching schemes available. The implementation currently supports the DrellYan $\left(Z / \gamma^{*}\right)$ process, and Higgs production via gluon-fusion (in the large top-mass limit) and is available for the dipole shower $[10,18]$. For the Drell-Yan process, it is possible to use both the $\mathrm{MC}$ and $\mathrm{MC}_{\mathrm{DY}}$ variants of the $\mathrm{MC}$ scheme [19]. This module was validated against a previous, independent, implementation using the published DY results of Ref. [17] and was also used to simulate the first results for this method in Higgs production [20]. KrkNLO can be enabled by using

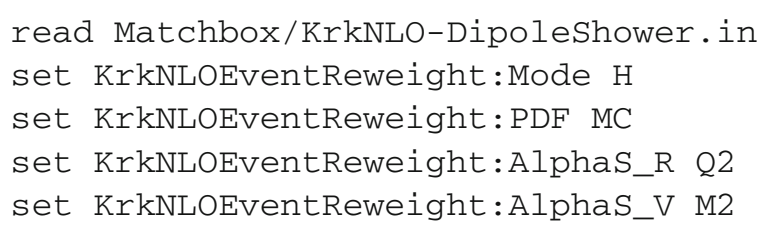

in combination with an MC-scheme PDF. The MC-scheme PDFs, example input-cards, and other relevant codes are hosted at https://krknlo.hepforge.org/.

\section{Parton shower developments}

\subsection{Angular-ordered parton shower}

A major restructuring of the angular-ordered parton shower has been performed in order to simplify the code, remove unused levels of abstraction and unused options. This is intended to improve the maintainability of the code and make new developments easier.

In addition we have changed the default interpretation of the ordering variable. When a final-state splitting $i \rightarrow j, k$ is generated, we can define the ordering scale in three different ways:

$$
\begin{aligned}
\tilde{q}^{2} & =\frac{q_{i}^{2}-m_{i}^{2}}{z(1-z)} ; \\
& =\frac{p_{T}^{2}+(1-z) m_{j}^{2}+z m_{k}^{2}-z(1-z) m_{i}^{2}}{z^{2}(1-z)^{2}} ; \\
& =\frac{2 q_{j} \cdot q_{k}+m_{j}^{2}+m_{k}^{2}-m_{i}^{2}}{z(1-z)} ;
\end{aligned}
$$

where $z$ is the light-cone momentum fraction carried by the particle $j, p_{T}$ is the transverse momentum of the splitting. When multiple emissions occur just one definition can be employed and this choice will also determine which quantity is preserved. We call this choice the "recoil scheme". By default, the scale is now expressed in terms of the dot-product of the emitted particles, i.e. Eq. (3), as discussed in Ref. [21]. We also include a veto on the masses of final-state jets, as suggested in Ref. [21], and we adopt the tuned parameter obtained in Ref. [21]. All of the choices for the interpretation of the evolution variable and tunes from Ref. [21] are available using the snippets

EvolutionScheme-*.in Tune-*.in

where * can be DotProduct-Veto, DotProduct, pT or Q2. This new recoil scheme, together with the veto on the final-state jets, allows a better description of the doublelogarithmically enhanced region, without overpopulating the tail of the distributions, as can be seen in Fig. 2 where the thrust distribution at the $\mathrm{Z}$ pole is compared to LEP data. The $q^{2}$-preserving scheme (blue) yields a good description of the tail, while the $p_{T}$-preserving (red) one performs better in the $T \approx 1$ region, however the dot-product-preserving scheme, together with the veto (green), gives the best agreement with data over the whole range.

\subsection{Spin correlations}

Herwig7 has always included spin correlations between production and decay of particles, and in both perturbative and non-perturbative decays. We have now completed the inclusion of spin correlations in all stages of the event generation by incorporating the correlations into both the angularordered and dipole parton showers. An example of these correlations is shown in Fig. 3 and this work is described in more detail in Ref. [23].

\subsection{Mass effects in the dipole shower}

We recall that Herwig 7 contains two shower algorithms, based on angular ordering (which we call QTilde) or dipole showering respectively. The dipole shower has been extended in version 7.1 to include the showering of top quarks in both their production and decay with the option to include the NLO correction to the decay. We show an example of the results for top production in Fig. 4, in comparison with ATLAS data [24]. The dipole shower can now perform showering of all Standard Model (SM) processes, including the NLO Powheg-type correction to all SM decays. The NLO correction can be switched on and off by setting,

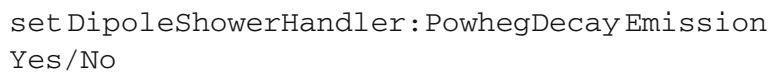

and is on by default.

We have also performed a detailed analysis and new derivation of the kinematics used to describe splittings of dipoles involving massive emitters and/or spectators. As part of this we have derived and implemented covariant formu- 

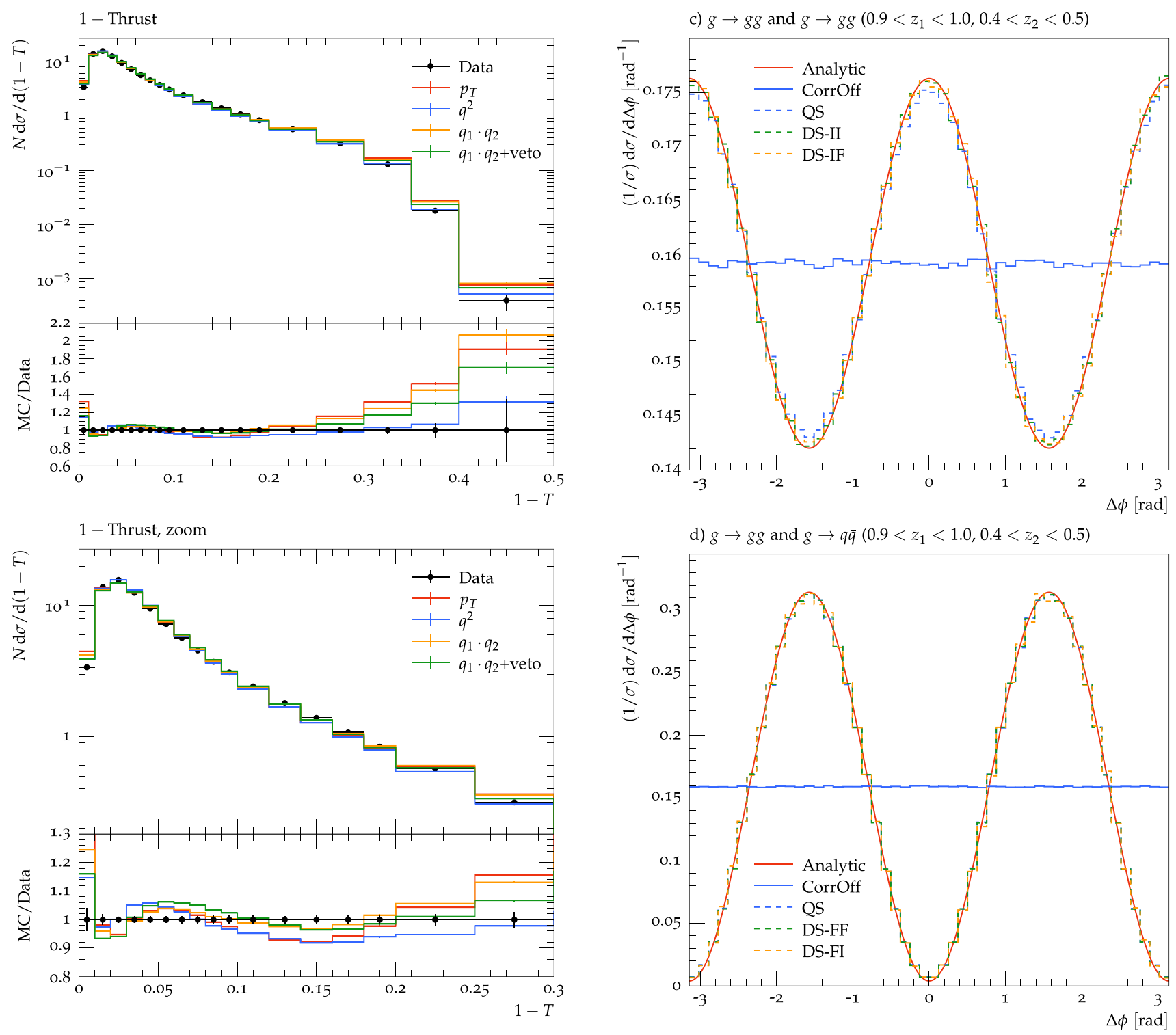

Fig. 2 The thrust at the Z-pole compared with data from the DELPHI [22] experiment. In the right panel a zoom for small $1-T$ values is shown

Fig. 3 Examples of the spin correlations in the parton shower for $g \rightarrow$ $g g$ with subsequent $g \rightarrow g g$ and $g \rightarrow q \bar{q}$ branching. For details, see Ref. [23]

\subsection{Shower variations and reweighting}

lations of the physical momenta of the partons following a splitting in terms of the physical momenta of the partons prior to the splitting for these dipoles. The kinematics for all dipole splittings in the dipole shower and Matchbox now use such a formulation, with an evolution variable which is directly connected to the transverse momentum variable relevant for the collinear or quasi-collinear limits. The effect of these improvements can be clearly seen in our modelling of $B$-Fragmentation in $e^{+} e^{-}$annihilation at the $Z^{0}$ mass, see Fig. 5, and more details will be covered in a forthcoming publication.
Evaluation of shower uncertainties is an important part of modern Monte Carlo studies. Shower uncertainties are traditionally evaluated by performing a full set of event simulations for each variation of interest.

To reduce the computational cost of evaluating shower uncertainties we have introduced functionality to perform on-the-fly parton shower reweighting in Herwig [27]. In this framework, each event is showered using a central set of parameters. In addition, on a splitting-by-splitting basis, we evolve a weight relative to the central shower for each set of varied parameters. We have currently implemented reweight- 

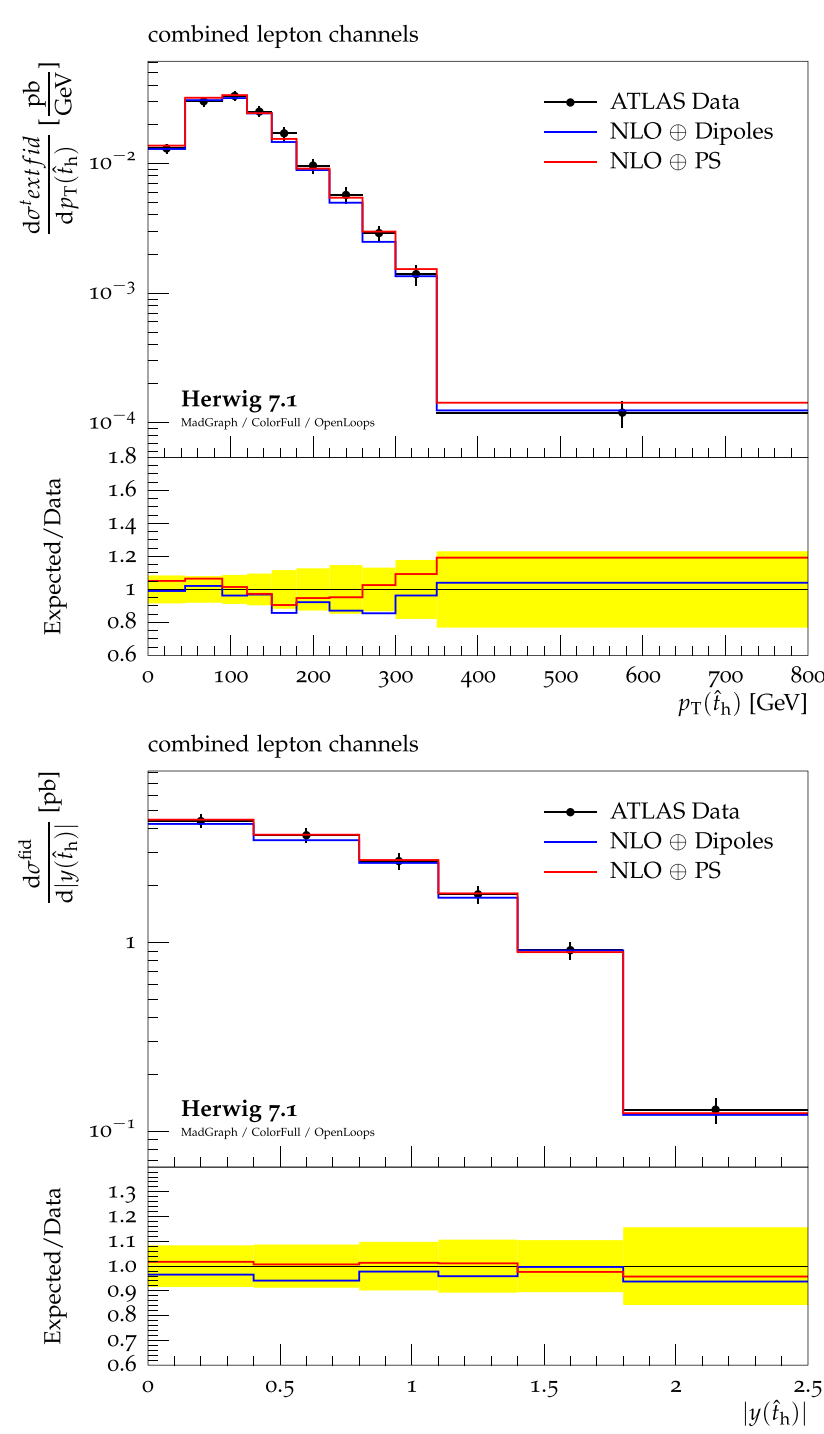

Fig. 4 Properties of top pair production in $7 \mathrm{TeV}$ collisions at the LHC, as measured by ATLAS [24] and predicted by the QTilde and Dipole showers using the NLO+PS setup of Matchbox in Herwig 7.1. More details will be presented in a forthcoming publication [25]. For these results we have used our run-time interfaces to MadGraph5_aMCatNLO [14] and OpenLoops [15] to evaluate scattering amplitudes for each phase space point, and ColorFull [16] to perform the colour algebra

ing to evaluate variations of the factorization and renormalization scales used in the shower however it is a general technique that could be applied to other variations in future developments.

A very efficient sequence of the veto algorithm for the central scale choice can lead to inefficient performance of the algorithm for the variations. We have included a 'detuning parameter' which can be used to improve the convergence of the reweighted results at the expense of a less efficient algorithm for the central prediction.

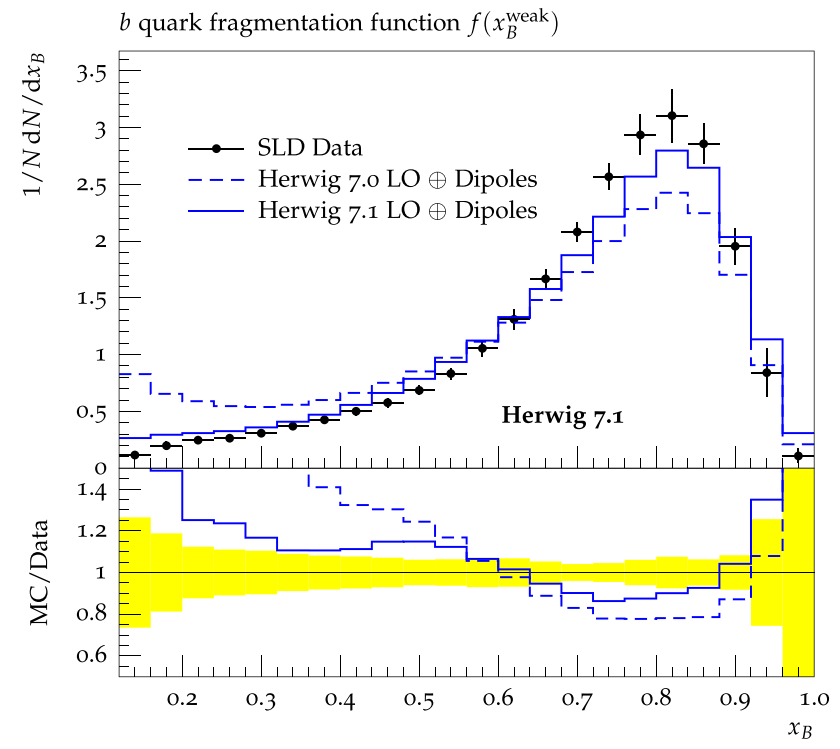

Fig. 5 The $B$-fragmentation as measured by SLD [26] and predicted by the dipole shower with the improved kinematics for massive quarks. More details will be presented in a forthcoming publication [25]

Reweighting is available in both showers. Multiple variations can be included in a single run and each variation requires a unique name, 'varName', which is used to identify the weight in the HepMC record. Each variation corresponds to a pair of scale factors, $\xi_{R}$ and $\xi_{F}$, to be applied to the renormalization and factorization scales respectively. Finally each variation can be applied to the showering of the hard process only (Hard), secondary processes only (Secondary) or to both parts (All):

\section{do ShowerHandler:AddVariation VarName $\mathrm{xR}$ xF Hard/Secondary/All set SplittingGenerator:Detuning Factor \\ do DipoleShowerHandler:AddVariation VarName xiR xiF Hard, Secondary, All set DipoleShowerHandler: Detuning Factor}

On top of using reweighting for the shower variations, the dipole shower offers a number of reweighting and biasing facilities which are e.g. used for the KrkNLO method (see above). These are available through the DipoleSplittingReweight and DipoleEvent Reweight classes. Very flexible veto functionality is also available for the angular ordered shower through the ShowerVeto and FullshowerVeto classes.

\subsection{Colour matrix element corrections}

General colour matrix element corrections for the dipole shower as presented in [28] and earlier outlined in [29] are 
now available in the new release. The colour matrix element corrections change the radiation pattern of the dipole shower for subsequent emissions by including a correction factor

$w_{i j, k}=-\frac{\operatorname{Tr}\left[\mathbf{T}_{i j} \cdot \mathbf{T}_{k} \mathbf{M}_{n}\right]}{\mathbf{T}_{i j}^{2} \operatorname{Tr}\left[\mathbf{M}_{n}\right]}$

along with each dipole splitting kernel $V_{i j, k}$, where $\mathbf{M}_{n}$ is the $n$-parton 'colour density operator' initialized by the amplitude and conjugate amplitude vectors at the level of the hard process which is evolved to higher multiplicities using the soft-collinear approximation. They can be enabled using the dipole shower with any of the Matchbox generated processes and the snippet

Matchbox/CMEC.in

\section{MPI model}

In order to simulate minimum-bias collisions, we use a 'dummy' matrix element to extract a first pair of partons with no transverse momentum from the colliding hadrons and describe all remaining interactions as (hard or soft) partonic scattering of the remnants. In this version, the processes handled by the ME are restricted to extract valence quarks only. The amount of forced splittings in the backward evolution to the incoming beams is therefore strongly reduced.

We have replaced the cross-section reweighter, which was previously used, and modified the matrix element used in minimum bias runs to reweight the cross section, such that the eikonalized cross sections are produced. This has the advantage of generating unit weights at the production level.

\subsection{Model for soft scatters}

Our model of soft interactions in the context of multiple partonic interactions (MPI) has been replaced by a different approach. The existing MPI model still forms the basis of the physics simulation by separating hard and soft interactions with the help of the parameter $p_{\perp}^{\min , 0}$ [30-32]. In the context of this framework a number of soft interactions $N_{\text {soft }}$ is determined as before.

Instead of the generation of a gluon pair for each soft interaction we now generate a number of rapidity-ordered gluons (and a pair of quarks) as depicted in Fig. 6. A first improvement of this model utilized multiperipheral kinematics [33], where the longitudinal momenta are slightly smeared, cf. [34].

The kinematics of the soft model have now been modified to use the algorithm described in [35], where the gluons are sampled randomly and flat in rapidity, resulting in a disappearance of the unphysical correlation found in [36]. Related

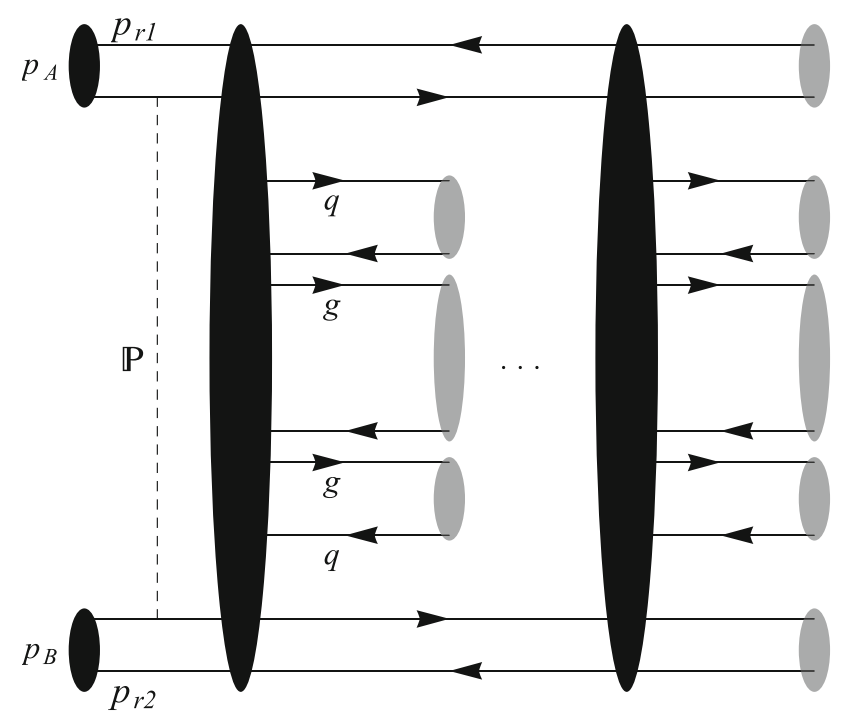

Fig. 6 Colour structure of soft particles produced with the new model for soft interactions, shown in the context of a complete hadron-hadron interaction

to the kinematics of the soft ladders is the distribution that is used to generate the transverse momenta. Here, we allow switching between different schemes and we found that it is beneficial to produce the hardest parton in the ladder according to the old distribution used in [2] and the rest of the partons flat below this maximal value.

The variable $p_{\perp}^{\mathrm{min}}$ which splits the hard from the soft scatterings was found to give a good description of data at high energies if a power law was used to parametrize the energy dependence. At small centre-of-mass energies $(\lesssim 200 \mathrm{GeV})$, this power-law generated values for $p_{\perp}^{\min }$ for which the eikonal model could not be solved. A comprehensive tuning effort showed that a power-law with an offset can be used to describe the data and solve the model at any sensible energy.

In order to complete the model towards softer and more forward interactions we also added a simple model for diffractive scattering which complements the hard MPI model for minimum-bias interactions. The model for diffractive final states heavily uses the cluster hadronization model already used by Herwig. Details of the model and several results have been presented elsewhere $[34,37,38]$. Here, we highlight two findings: most notably, the unphysical predictions for the distribution of forward rapidity gaps is now replaced by an excellent description of data, Fig. 7, highlighting the expected composition of non-diffractive events at small gap sizes, and diffractive contributions at large gaps. We stress the fact that the old model, which generated a 'bump' structure in this spectrum due to artificial colour reconnections, was not meant to describe these interactions, so no conclusions from this data comparison could be drawn. 


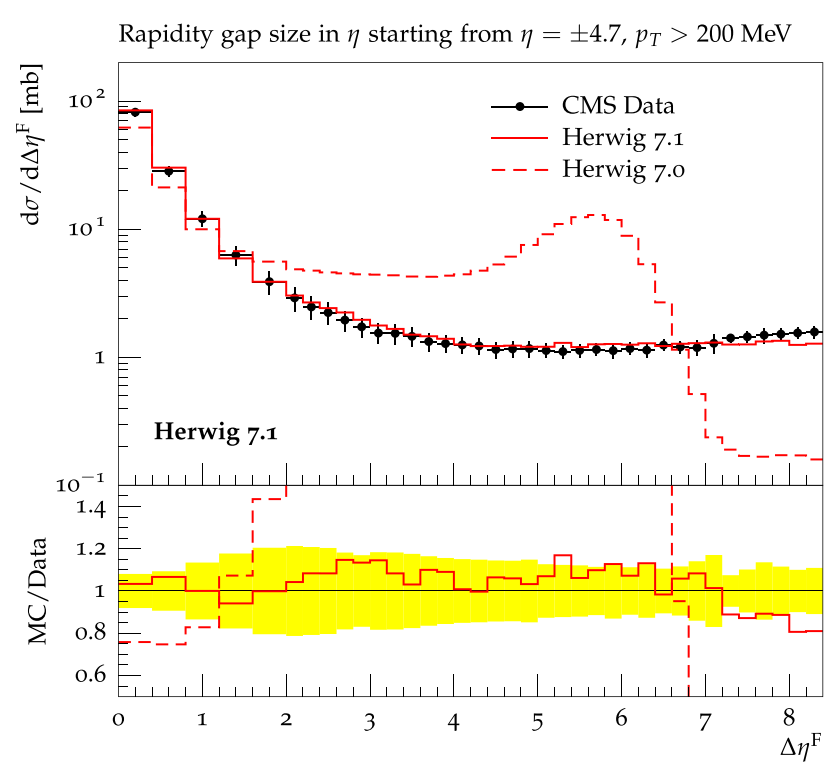

Fig. 7 The distribution of forward rapidity gaps with the new model including a model for diffractive final states (Herwig 7.1), compared to the old model (Herwig 7.0) and CMS data [39]

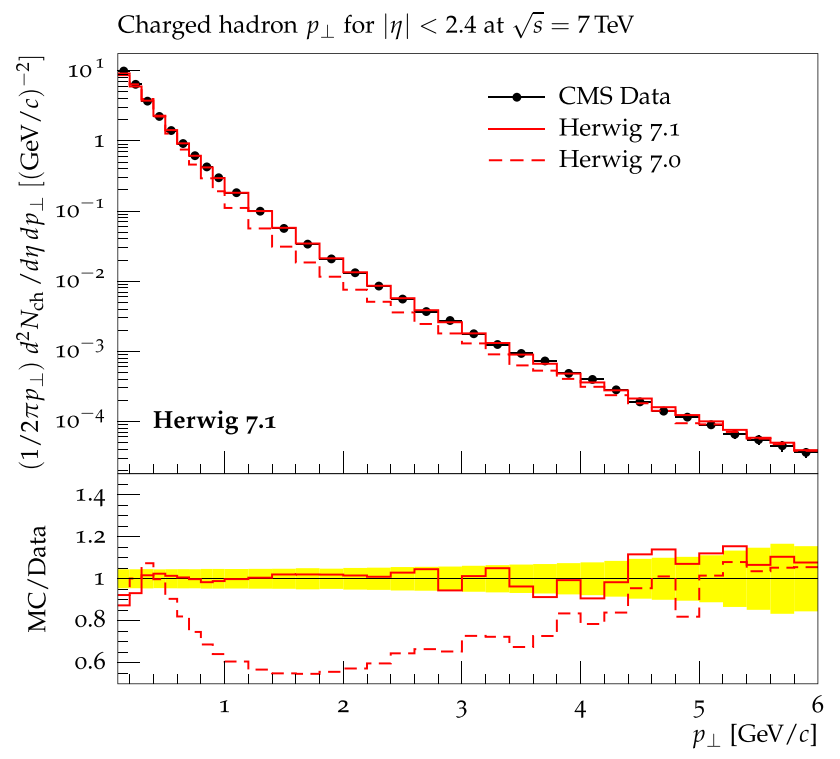

Fig. 8 Low transverse momentum spectrum of charged particles in non-single diffractive events with our old and new models for soft interactions compared to CMS data [40]

Another positive result of the new modelling of soft particle production is the improvement of soft transverse momentum spectra of charged particles, also in minimum-bias interactions, see Fig. 8.

The model for soft interactions has become the new default model. The matrix elements for diffractive scattering are used alongside the hard and soft MPI model by default in the simulation of minimum-bias matrix elements. There are two new parameters for the soft interaction model that determine the number of gluons per soft interaction and its growth with

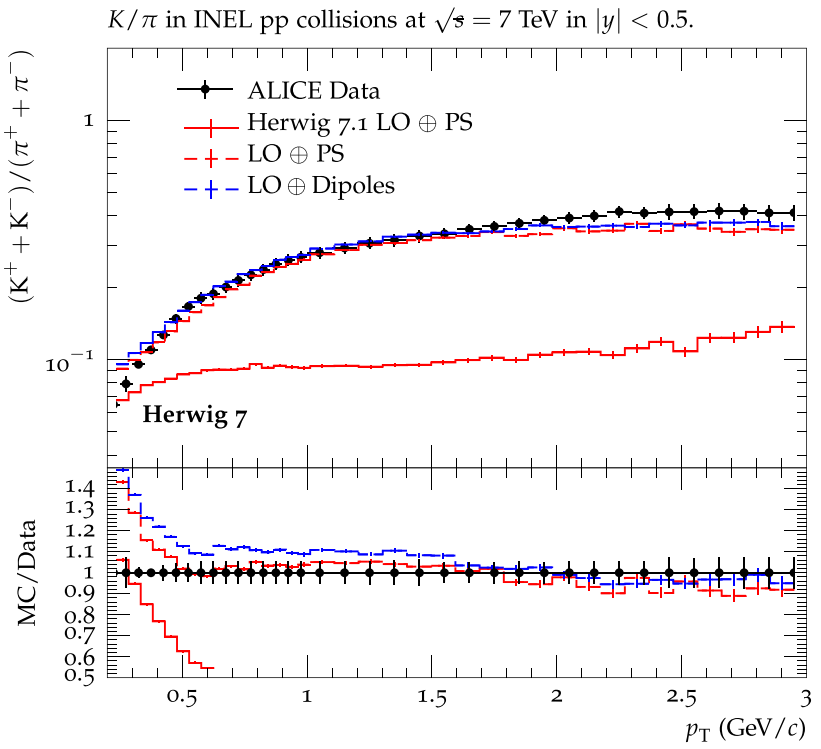

Fig. 9 The $\mathrm{K}$ to $\pi$ ratio in inelastic events in comparison with ALICE data [43]

energy. Both parameters have been tuned to minimum-bias data.

Another change that is more on the technical side is the introduction of the parameter that controls the ratio of the diffractive cross-section as part of the inelastic cross-section, named DiffractionRatio. It was previously a combination of the CSNorm parameter and the construction of the matrix element weight. The new parameter allows a more controlled and physically motivated tuning. Many of the changes that have been made to the handling of multiple parton interactions are described in detail in [41].

\subsection{Colour reconnection}

While the plain Colour Reconnection model [42] is an integral part of the description of general properties of Minimum Bias (MB) data, the description of flavour specific observables remained difficult. With Herwig 7.1.5 we introduced a new Colour Reconnection model that reconnects clusters based on geometrical properties. We also allow multiple mesonic clusters to form a baryonic type cluster if certain requirements are met. This gives an important lever on the baryon to meson ratio and proved to be a good starting point for the description of flavour observables. Additionally we allow non-perturbative $g \rightarrow s \bar{s}$ splitting for an additional source of strangeness. With the new model, the whole range of MB data can be described with similarly good quality and the description of hadronic flavour observables improves significantly. An example of the strangeness production is shown in Fig. 9, where we see a greatly improved description of ALICE data with either of the shower models. 


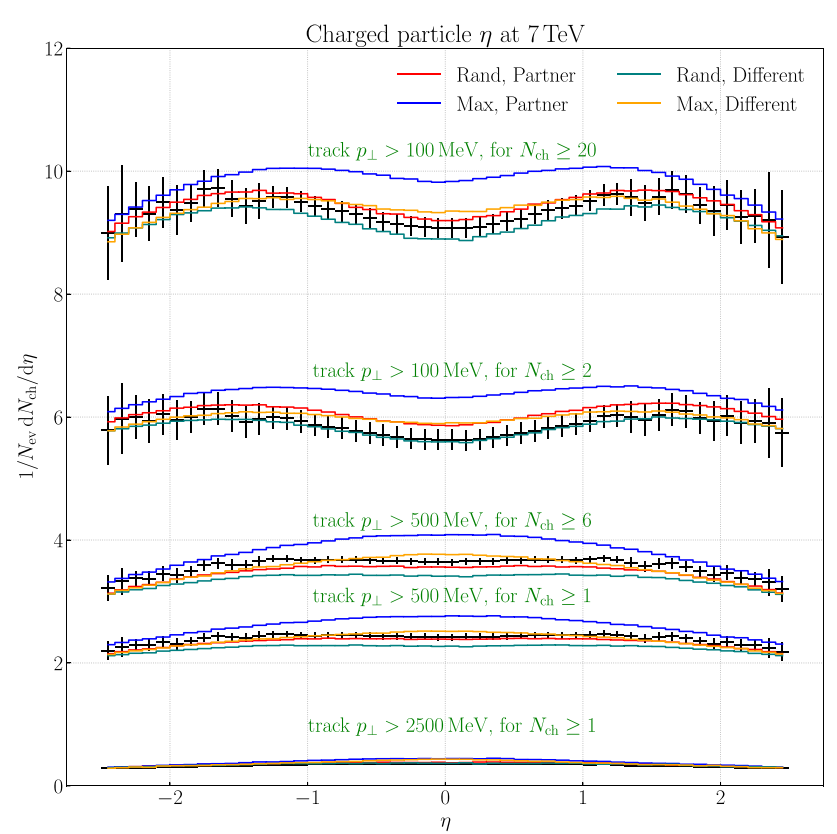

Fig. 10 The charged-particle multiplicity is plotted against the rapidity for multiple cuts (green labels) on the hardest track transverse momentum and number of charged particles. Data is taken from [46]. This observable is sensitive to the choices that are employed as the starting conditions of the parton shower process. The four choices are described in Sec. 4.3. While height differences are easily modified in the tuning process, shape differences prefer choices with a random colour partner for gluons in the hard process

For more details on the implementation and the details of the model, we refer to [44]. Please note that the spacetime picture related model [45] is not included in the current release.

\subsection{Further modifications of the MPI model}

It was found that changed starting conditions for the showering of the gluons, in particular the recoil partner and scale choice, are beneficial for the description of charged multiplicities over rapidity. The default choice is the same as used previously in the showering of NLO matched samples and external LHE files. In Fig. 10 we illustrate the effect for the choices that choose the evolution partner randomly (Rand) or according to the maximal angle (Max) and allow the shower starting scale choice to be chosen according to the partner (Partner) or differently (Different).

The combination of all the changes described here and in the previous subsections required a retuning of the MPI model. Details are outlined in [41].

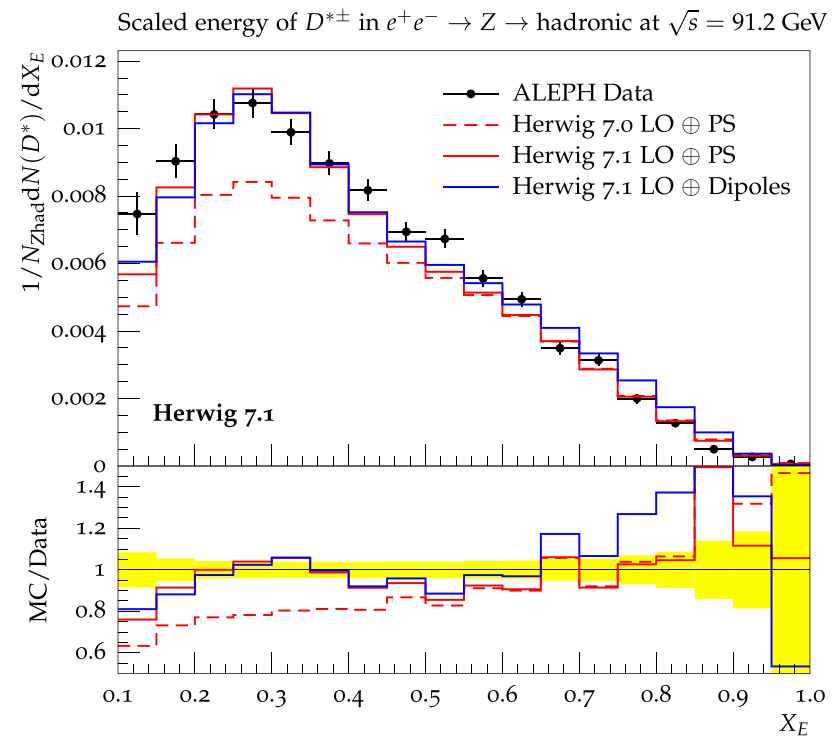

Fig. 11 The spectrum of $D^{*}$ mesons measured by the ALEPH experiment [48] compared to Herwig. As an example, we show LO plus PS predictions, however as expected these are not significantly changed in the presence of higher order corrections

\section{Decays}

\subsection{EvtGen interface}

The internal Herwig modelling of hadron decays includes sophisticated modelling with off-shell effects and spin correlations. However, it has proven impossible to provide a good tune to data for the decay of bottom and charm mesons, largely due to the lack of published distributions. Given that EvtGen[47] has been tuned to non-public data from the Bfactory experiments and internally uses similar algorithms to include spin correlations in particle decays, in Herwig 7.1 we include an interface to EvtGen which communicates the spin information between the two programs ensuring that the full correlations are generated. EvtGen is now the default for the decay of bottom and charm mesons. As there is less data for bottom and charm baryons and our modelling of baryonic form-factors is more sophisticated, the decays of heavy baryons continue to be performed by Herwig. This leads to the improvement of a number of distributions, e.g. the momentum distribution of $D^{*}$ mesons [48], Fig. 11, where there is a significant contribution from $D^{*}$ mesons produced in bottom meson decays.

\subsection{Perturbative decays}

The classes implementing perturbative decays, in both the Standard Model and for BSM models, have been restructured. This allows the several previous implementations of hard radiation corrections in these decays, in both the 
POWHEG and matrix element correction schemes, to be combined and generalised. This now allows us to apply POWHEG-style hard corrections to a much wider range of decays, in particular in BSM models, and also include hard QED radiation. This restructuring also allows these decays, and the POWHEG corrections, to be used with both parton shower modules.

\section{BSM physics}

We have made significant improvements to the handling of models in the Universal FeynRules Output (UFO) format. Previously we could only handle vertices that had the perturbative form of the interaction, for example $\left(p_{1}-p_{2}\right)^{\mu}$ for vector-scalar-scalar interactions, where $p_{1,2}$ are the four momenta of the scalar particles.

We now make use of the sympy package [49] to allow us to write code capable of evaluating the HELAS building blocks for arbitrary Lorentz structures. This allows Herwig to be used to simulate a much wider class of BSM models with, for example, spin $\frac{3}{2}$ particles, colour flows involving $\varepsilon$ tensors and sextet particles, and many four-point interactions now supported. Splitting functions for the production of electromagnetic radiation are now also created by default for BSM particles.

\section{Other changes}

Besides the major physics improvements highlighted in the previous sections, we have also made a number of smaller changes to the code and build system which we will summarize below. Please refer to the online documentation for a fully detailed description or contact the authors.

\subsection{Steering, input files and weights}

The steering of the Herwig executable has seen a number of improvements, mainly:

- A new run mode has been added to solely perform the merging of integration grids from parallel integration runs,

\section{Herwig mergegrids <run file name>}

- A high-level run-time interface is now available to steer Herwig within more complex frameworks such as experimental software without the need to execute the binary. This includes all of the read, build, integrate, mergegrids and run steps.
The structure of input files for non-Matchbox-based processes has been adapted to use the snippet input file mechanism and is now in line with steering matched and merged processes. On top of this, a large number of input file switches which have before used On, off or True, False to indicate their state have been changed to Yes, No.

As far as integration and event generation are concerned, we have made a choice that by default sampling is run in AlmostUnweighted mode, i.e. events carry in general varying weights, most of which are unity. This is to account for the fact that the grid adaption might only have encountered a maximum weight close to the true maximum weight and strict unweighting in this case could skew distributions and cross section estimates. The reference weight to which events are unweighted can also be adjusted to keep weight distributions mostly narrow while reducing fluctuations in tails due to a small frequency of contributing events. Alongside this, the adaption parameters of both the CellGridSampler and Monacosampler have been revised.

\subsection{SaS parton distribution functions}

As version 6 of the LHAPDF [50] package does not contain any parton distributions for the partons inside resolved photons the FORTRAN code and an interface to the SchulerSjöstrand [51] parton distribution functions for the photon have been included to allow the simulation of resolved photon processes.

\section{$7.3 \mathrm{FxFx}$}

The FxFx merging module was introduced in [1] to provide support of the NLO multi-jet merging method of [52], via Les Houches-accord event (LHE) files generated by MadGraph 5 /aMc@NLO [14].

In Herwig 7.2 this functionality is available by default, being compiled with the main part of the code. The framework also provides an interface for merging of tree-level events generated either by MadGraph 5/aMCQNLO or AlpGen via the MLM technique [53,54], replacing all the functionality that first appeared in [6]. The relevant input files for the FxFx merging and tree-level merging are now LHE-FXFx. in and LHE-MGMerging. in respectively. We emphasize that it is essential to include the MC@NLO matching settings for MadGraph 5/aMC@NLO when performing the FxFx merging, as given in LHE-MCatNLO . in. These settings should not be included when merging treelevel events. The tree-level merging functionality via MadGraph 5/aMC@NLO events uses the event tags in the appropriately-generated LHE files and requires the option MergeMode to be set to TreeMG5, as is done by default in LHE-MGMerging. in. To enable merging with events 
generated via AlpGen, MergeMode should be switched to Tree.

We note that the FxFx functionality has been tested thoroughly only for $\mathrm{W}+$ jets and $\mathrm{Z}+$ jets events in [55], where it was compared against LHC data at 7 and $8 \mathrm{TeV}$. We also note that no tuning was performed in Herwig using events generated via this interface.

\subsection{Default PDF}

The default parton distribution function has been changed from that of MMHT 2014 [56] to CT14 [57].

\subsection{Minor improvements and bug fixes}

A number of minor changes and bug fixes are worth noting, in particular, there have been new options for the physics simulation besides the ones described in the previous text:

- Colour reconnection of octet systems into a single cluster are now prevented, improving the description of a number of observables sensitive to these dynamics, as well as some unexpected features which have been observed in preceeding work [58].

- For both showers it is now possible to alter the scale choice and ordering properties in $g \rightarrow q \bar{q}$ splittings.

- New options of shower scale choices are available for NLO matched processes.

- The $\alpha_{s}$ running in the dipole shower can now explicitly be switched to use the CMW scheme [59], through both a scaling of its argument as well as by explicitly adding the $\alpha_{s}^{2} K_{g}$ contribution, such that these contributions do not anymore need to be absorbed into a tuned value of $\alpha_{s}$.

- Several options have been added for the emission phase space in the dipole shower, which are subject to a more detailed, future study.

- Structures in ThePEG have been extended to cover processes which do not exhibit a (tree) diagram-like internal structure, such as instanton- and sphaleron-induced transitions.

- major updates in the Tests directory to improve both the generation of input files and add new Rivet analyses.

- a number of changes have been made to ensure that the Herwig code compiles with the Intel and Clang compilers. A number of changes have also been made to ensure compilation with recent gcc compilers, including gcc9.

- The deprecated UA5 soft underlying event model has been removed.

- The input files for a number of old tunes have been removed.

- The cut-off for photon radiation from leptons has been reduced to $10^{-6} \mathrm{GeV}$.
- Support for fixed target collisions has been included, together with an example input file.

- The analytic calculation of the partial width for $V \rightarrow S S$ decays has been corrected.

- The setting of masses in UFO models where one parameter sets the masses of many particles has been fixed.

- An effective vertex for the processes $h^{0} \rightarrow Z^{0} \gamma$ has been added so the $Z^{0}$ mass is correctly generated in this decay.

- Fix to the MEvv2vs class so that more than one fourpoint vertex is allowed.

- A missing $t$-channel diagram has geen added to the MEfv2fs class.

- Changes to avoid 0/0 have been made in the VVVDecayer class.

- An option to use the internal Standard Model Higgs boson vertices for UFO models which do not implement the full Higgs sector has been added.

- Several bugs in the presence of spacelike off-shell incoming legs have been fixed in ThePEG's StandardxComb and Herwig's Tree2toNPhasespace classes.

- The option of an asymetric splitting of the colour flows for the $g \rightarrow g g$ branching in the dipole shower has been added.

- Additional kernels are implemented for the $\tilde{q}$ shower to incorporate the Catani-Marchesini-Webber (CMW) scheme as part of a linear scheme. By default, the scheme is absorbed in a change of the nominal value of the strong coupling $\alpha_{S}\left(M_{Z}\right)$. A similar scheme has been available for the dipole shower since Herwig 7.1.

- The dipole shower has been tuned using the method described in [60].

Technical issues which have been addressed include:

- Matchbox is now able to handle processes which do not contain coloured external legs.

- The dipole shower can handle zero-momentum-transfer initial-final colour connections, which have prevented running minimum bias simulation with this shower algorithm before.

- Several levels of assumptions (such as Standard Modellike interactions, conservation of lepton flavour number, quark flavour diagonal interactions) can be imposed on the generation of candidate sub-processes to reduce combinatorial complexity for processes with many legs.

- The old ClassTraits mechanism used by ThePEG has been replaced by the new DescribeClass mechanism consistently in all the Herwig code.

- Changes to the templates for dimension-full quantities to improve the maintainability of this code. Regrettably this is incompatible with gcc 4.8 and therefore gcc 4.9 is now the oldest supported version of gcc. 
- A number of changes have been made to ensure the bootstrap script works with python3, however a number of our dependencies do not yet support python 3 and therefore the code still uses python2.

- The generation of trial values of the scale and light-cone momentum fraction in the angular-ordered parton shower has been restructured to improve performance.

- The calculation of the cross section in Matchbox processes has been restructured to reduce calls to the parton distribution functions, and hence improve performance.

- Changes have been made to improve the detection of recent boost versions at compile time.

\subsection{Build and external dependencies}

Since version 7.1, Herwig has enforced the use of a $\mathrm{C}++11$ compliant compiler, and $\mathrm{C}++11$ syntax and standard library functionality is used widely within the code. The herwig-bootstrap script is able to provide such a compiler along with a full Herwig plus dependencies build. herwig-bootstrap will also enforce the newest versions of external amplitude providers; specifically we now use:

- OpenLoops [15] versions $\geq 2.0 .0$ with the Collier library [61] for tensor reduction (should older versions of OpenLoops be required, the input files require the additionaloption set OpenLoops:UseCollier Off), and

- GoSam versions $\geq 2.0 .4$ to pick up the correct normalization for loop induced processes outside of specialized setups.

A number of changes have also been implemented to reduce run-time load for allocating and de-allocating various containers, and to reduce overall memory consumption.

\section{Example results}

Herwig 7.2 has been thoroughly validated against a wide range of existing data, as implemented in the Rivet and FastJet frameworks $[62,63]$. Parameter tuning has been performed using Professor [64].

Here, we illustrate some examples of the fact that we can simulate LHC events with any combination of LO or NLO matrix elements, matched with the angular-ordered or dipole showers using either additive (MC@NLO-like) or multiplicative (POWHEG-like) methods, as well as multi-jet merging, for $\mathrm{Z}$ boson production. In Fig. 12, we show the results in comparison with ATLAS data [65].

The upper plot shows that, as would be hoped, merging with multi-jet matrix elements enables a good description of the data over a wide range of jet mupliticities. The lower
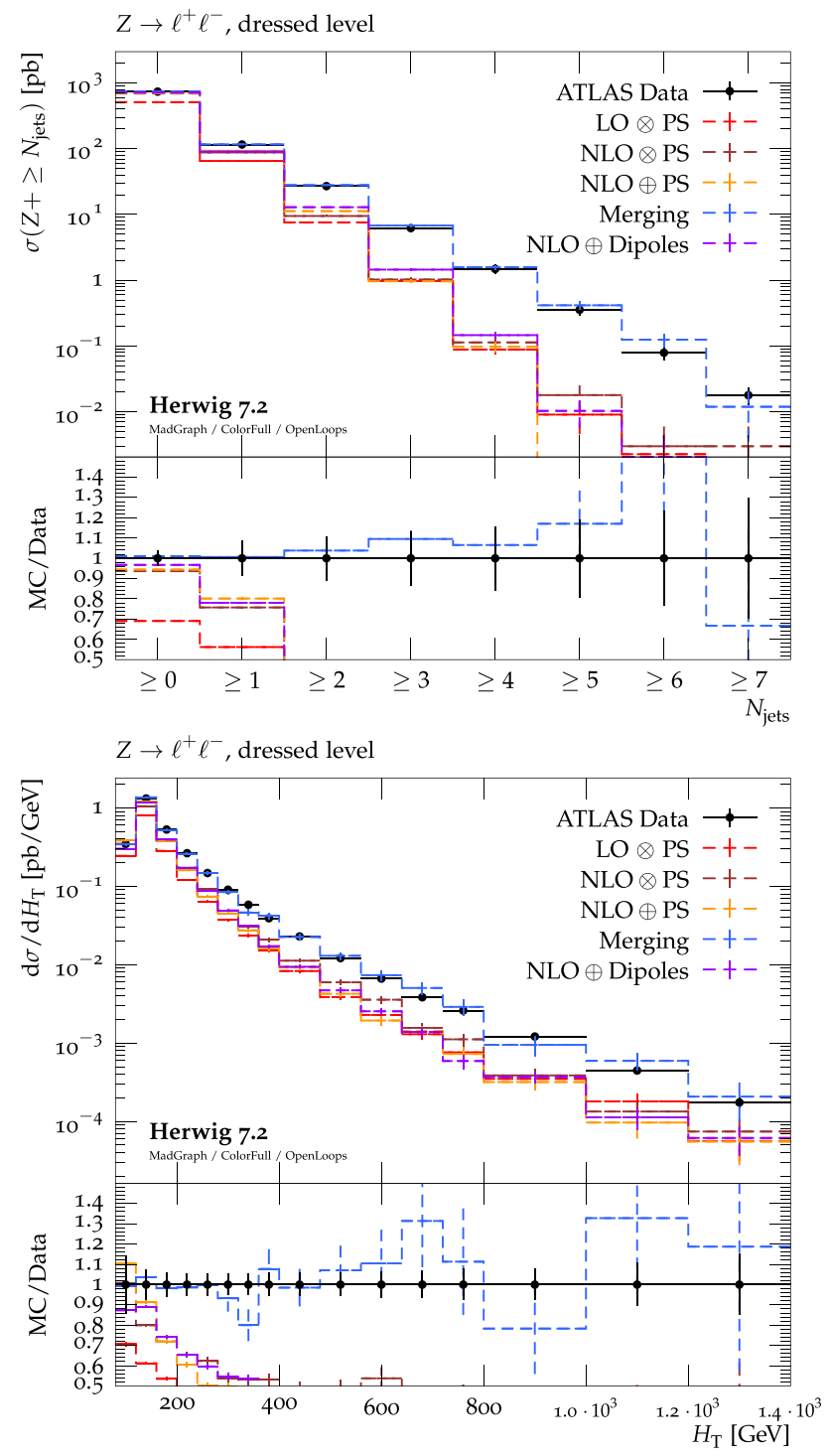

Fig. 12 The cross section for $\mathrm{Z}$ production in association with $N_{\text {jets }}$ jets (upper) or differentially with respect to the total scalar sum of final state transverse momenta, $H_{\mathrm{T}}$, (lower) in comparison with ATLAS data [65]

plot shows that even for more inclusive quantities, such as the total scalar transverse momentum, the multijet effects are important.

A wide range of further plots can be found at https:// herwig.hepforge.org/plots/herwig7.2.

\section{Summary and outlook}

We have described a new release, version 7.2, of the Herwig event generator. This new release contains a number of improvements to both perturbative and non-perturbative sim- 
ulation of collider physics and will form the basis of further improvements to both physics and technical aspects.

Acknowledgements We are indebted to Leif Lönnblad for his authorship of ThePEG, on which Herwig is built, and his close collaboration, and also to the authors of Rivet and Professor. We are also grateful to Malin Sjödahl for providing the ColorFull library for distribution along with Herwig. This work was supported in part by the European Union as part of the FP7 and H2020 Marie Skłodowska-Curie Initial Training Networks MCnetITN and MCnetITN3 (PITN-GA-2012-315877 and 722104) and the UK Science and Technology Facilities Council (Grant numbers ST/P000800/1, ST/P001246/1). JB and CR are part of the MorePheno project that is funded by the European Research Council (ERC) under the European Union's Horizon 2020 research and innovation programme, Grant agreement No 668679. AP acknowledges support by the ERC Grant ERC-STG-2015-677323. GB thanks the UK Science and Technology Facilities Council for the award of a studentship. AS acknowledges support from the National Science Centre, Poland Grant No. 2016/23/D/ST2/02605. This work has been supported by the BMBF under Grant number 05H18VKCC1 and also by the Czech Science Foundation (GACR) Grant 18-07846Y.

Data Availability Statement This manuscript has no associated data or the data will not be deposited. [Authors' comment: The simulation code is publicly available from https://herwig.hepforge.org and the plots and data files generated in the production of this paper can be found at https://herwig.hepforge.org/plots/herwig7.2/index.html.]

Open Access This article is licensed under a Creative Commons Attribution 4.0 International License, which permits use, sharing, adaptation, distribution and reproduction in any medium or format, as long as you give appropriate credit to the original author(s) and the source, provide a link to the Creative Commons licence, and indicate if changes were made. The images or other third party material in this article are included in the article's Creative Commons licence, unless indicated otherwise in a credit line to the material. If material is not included in the article's Creative Commons licence and your intended use is not permitted by statutory regulation or exceeds the permitted use, you will need to obtain permission directly from the copyright holder. To view a copy of this licence, visit http://creativecomm ons.org/licenses/by/4.0/.

Funded by SCOAP ${ }^{3}$.

\section{References}

1. J. Bellm et al., Herwig 7.0/Herwig++ 3.0 release note. Eur. Phys. J. C 76, 196 (2016). https://doi.org/10.1140/epjc/ s10052-016-4018-8. arXiv:1512.01178

2. M. Bähr et al., Herwig++ physics and manual. Eur. Phys. J. C 58, 639-707 (2008). https://doi.org/10.1140/epjc/ s10052-008-0798-9. arXiv:0803.0883

3. M.Bähr et al., Herwig++ 2.2 release note. arXiv:0804.3053

4. M.Bähr et al., Herwig++ 2.3 release note. arXiv:0812.0529

5. S. Gieseke et al., Herwig++ 2.5 release note. arXiv:1102.1672

6. K. Arnold et al., Herwig++ 2.6 release note. arXiv:1205.4902

7. J. Bellm et al., Herwig++ 2.7 release note. arXiv: 1310.6877

8. J. Bellm et al., Herwig 7.1 release note. arXiv:1705.06919

9. J. Bellm, S. Gieseke, S. Plätzer, Merging NLO multi-jet calculations with improved unitarization. arXiv: 1705.06700

10. S. Plätzer, S. Gieseke, Dipole showers and automated NLO matching in Herwig++. Eur. Phys. J. C 72, 2187 (2012). https://doi.org/ 10.1140/epjc/s10052-012-2187-7. arXiv:1109.6256
11. S. Plätzer, Controlling inclusive cross sections in parton shower + matrix element merging. JHEP 08, 114 (2013). https://doi.org/10. 1007/JHEP08(2013)114. arXiv:1211.5467

12. ATLAS collaboration, G. Aad et al., Measurement of the transverse momentum distribution of $\mathrm{Z} / \gamma^{*}$ bosons in proton-proton collisions at $\sqrt{s}=7 \mathrm{TeV}$ with the ATLAS detector, Phys. Lett. B705 (2011) 415-434. https://doi.org/10.1016/j.physletb.2011.10. 018. arXiv: 1107.2381

13. ATLAS collaboration, G. Aad et al., Study of jets produced in association with a $W$ boson in $p p$ collisions at $\sqrt{s}=7 \mathrm{TeV}$ with the ATLAS detector, Phys. Rev. D 85 (2012) 092002. https://doi. org/10.1103/PhysRevD.85.092002. arXiv:1201.1276

14. J. Alwall et al., The automated computation of tree-level and nextto-leading order differential cross sections, and their matching to parton shower simulations. JHEP 07, 079 (2014). https://doi.org/ 10.1007/JHEP07(2014)079. arXiv:1405.0301

15. F. Cascioli, P. Maierhöfer, S. Pozzorini, Scattering amplitudes with open loops. Phys. Rev. Lett. 108, 111601 (2012). https://doi.org/ 10.1103/PhysRevLett.108.111601. arXiv:1111.5206

16. M. Sjödahl, ColorFull-a C++ library for calculations in $\mathrm{SU}(\mathrm{Nc})$ color space. Eur. Phys. J. C 75, 236 (2015). https://doi.org/10.1140/ epjc/s10052-015-3417-6. arXiv:1412.3967

17. S. Jadach, W. Płaczek, S. Sapeta, A. Siódmok, M. Skrzypek, Matching NLO QCD with parton shower in Monte Carlo scheme: the KrkNLO method. JHEP 10, 052 (2015). https://doi.org/10.1007/ JHEP10(2015)052. arXiv:1503.06849

18. S. Plätzer, S. Gieseke, Coherent parton showers with local recoils. JHEP 01, 024 (2011). https://doi.org/10.1007/JHEP01(2011)024. arXiv:0909.5593

19. S. Jadach, W. Płaczek, S. Sapeta, A. Siódmok, M. Skrzypek, Parton distribution functions in Monte Carlo factorisation scheme. Eur. Phys. J. C 76, 649 (2016). https://doi.org/10.1140/epjc/ s10052-016-4508-8. arXiv:1606.00355

20. S. Jadach, G. Nail, W. Płaczek, S. Sapeta, A. Siódmok, M. Skrzypek, Monte Carlo simulations of Higgs-boson production at the LHC with the KrkNLO method. Eur. Phys. J. C 77, 164. https://doi.org/10.1140/epjc/s10052-017-4733-9. arXiv:1607.06799

21. G. Bewick, S. Ferrario Ravasio, P. Richardson, M.H. Seymour, Logarithmic accuracy of angular-ordered parton showers. arXiv:1904.11866

22. DELPHI collaboration, P. Abreu et al., Tuning and test of fragmentation models based on identified particles and precision event shape data. Z. Phys. C 73, 11-60 (1996). https://doi.org/10.1007/ s002880050295

23. P. Richardson, S. Webster, Spin correlations in parton shower simulations. arXiv: 1807.01955

24. ATLAS collaboration, G. Aad et al., Differential top-antitop crosssection measurements as a function of observables constructed from final-state particles using pp collisions at $\sqrt{s}=7 \mathrm{TeV}$ in the ATLAS detector, JHEP 06, 100 (2015). https://doi.org/10.1007/ JHEP06(2015)100. arXiv:1502.05923

25. K. Cormier, S. Gieseke, S. Plätzer, C. Reuschle, P. Richardson, $\mathrm{S}$. Webster, Systematics and uncertainties of top pair production at $\mathrm{NLO}+\mathrm{PS}$

26. SLD collaboration, K. Abe et al., Measurement of the b quark fragmentation function in Z0 decays. Phys. Rev. D 65, 092006 (2002). https://doi.org/10.1103/PhysRevD.66.079905. https://doi. org/10.1103/PhysRevD.65.092006. arXiv:hep-ex/0202031

27. J. Bellm, S. Plłtzer, P. Richardson, A. Siódmok, S. Webster, Reweighting parton showers. Phys. Rev. D 94, 034028 (2016). https://doi.org/10.1103/PhysRevD.94.034028. arXiv:1605.08256

28. S. Plätzer, M. Sjödahl, J. Thorén, Color matrix element corrections for parton showers. JHEP 11, 009 (2018). https://doi.org/10.1007/ JHEP11(2018)009. arXiv:1808.00332 
29. S. Plätzer, M. Sjödahl, Subleading $N_{c}$ improved parton showers. JHEP 07, 042 (2012). https://doi.org/10.1007/JHEP07(2012)042. arXiv: 1201.0260

30. M. Bähr, S. Gieseke, M.H. Seymour, Simulation of multiple partonic interactions in Herwig++. JHEP 07, 076 (2008). https://doi. org/10.1088/1126-6708/2008/07/076. arXiv:0803.3633

31. M. Bähr, J.M. Butterworth, M.H. Seymour, The underlying event and the total cross section from tevatron to the LHC. arXiv:0806.2949

32. M.Bähr, J.M. Butterworth, S. Gieseke, M.H. Seymour, Soft interactions in Herwig++. arXiv:0905.4671

33. M. Baker, K.A. Ter-Martirosian, Gribov's Reggeon calculus: its physical basis and implications. Phys. Rept. 28, 1-143 (1976). https://doi.org/10.1016/0370-1573(76),90002-8

34. S. Gieseke, F. Loshaj, P. Kirchgaeßer, Soft and diffractive scattering with the cluster model in Herwig. Eur. Phys. J. C 77, 156 (2017). https://doi.org/10.1140/epjc/s10052-017-4727-7. arXiv: 1612.04701

35. S. Jadach, Rapidity generator for Monte-Carlo calculations of cylindrical phase space. Comput. Phys. Commun. 9, 297-304 (1975). https://doi.org/10.1016/0010-4655(75),90081-8

36. M. Azarkin, P. Kotko, A. Siódmok, M. Strikman, Studying minijets and MPI with rapidity correlations. Eur. Phys. J. C 79, 180 (2019). https://doi.org/10.1140/epjc/s10052-019-6670-2. arXiv: 1806.09016

37. S. Gieseke, P. Kirchgaeßer, F. Loshaj, Soft interactions in Herwig, in 8th International Workshop on Multiple Partonic Interactions at the LHC (MPI@LHC 2016) San Cristobal de las Casas, Chiapas, Mexico, November 28-December 2, 2016, 2017. arXiv:1703.10808

38. S. Gieseke, P. Kirchgaeßer, F. Loshaj, A new model for soft interactions in Herwig, in 23rd Cracow Epiphany Conference on Particle Theory Meets the First Data from LHC Run 2 Cracow, Poland, January 9-12, 2017, 2017. arXiv:1704.00911

39. CMS collaboration, V. Khachatryan et al., Measurement of diffraction dissociation cross sections in pp collisions at $\sqrt{s}=7 \mathrm{TeV}$, Phys. Rev. D 92, 012003 (2015). https://doi.org/10.1103/PhysRevD.92. 012003. arXiv: 1503.08689

40. CMS collaboration, V. Khachatryan et al., Transverse-momentum and pseudorapidity distributions of charged hadrons in $p p$ collisions at $\sqrt{s}=7$ TeV. Phys. Rev. Lett. 105, 022002 (2010).https:// doi.org/10.1103/PhysRevLett.105.022002. arXiv:1005.3299

41. J. Bellm, S. Gieseke, P. Kirchgaeßer, Improving the description of multiple interactions in Herwig. arXiv:1911.13149

42. S. Gieseke, C. Röhr, A. Siódmok, Colour reconnections in Herwig++. Eur. Phys. J. C 72, 2225 (2012). https://doi.org/10.1140/ epjc/s10052-012-2225-5. arXiv:1206.0041

43. ALICE collaboration, J. Adam et al., Measurement of pion, kaon and proton production in proton-proton collisions at $\sqrt{s}=7$ TeV. Eur. Phys. J. C 75, 226 (2015). https://doi.org/10.1140/epjc/ s10052-015-3422-9. arXiv:1504.00024

44. S. Gieseke, P. Kirchgaeßer, S. Plätzer, Baryon production from cluster hadronisation. Eur. Phys. J. C 78, 99 (2018). https://doi. org/10.1140/epjc/s10052-018-5585-7. arXiv:1710.10906

45. J. Bellm, C.B. Duncan, S. Gieseke, M. Myska, A. Siódmok, Spacetime colour reconnection in Herwig 7, accepted for publication in Eur. Phys. J. C (2019). arXiv:1909.08850

46. ATLAS collaboration, G. Aad et al., Charged-particle multiplicities in $\mathrm{pp}$ interactions measured with the ATLAS detector at the LHC. New J. Phys. 13, 053033 (2011). https://doi.org/10.1088/ 1367-2630/13/5/053033. arXiv:1012.5104

47. D.J. Lange, The EvtGen particle decay simulation package. Nucl. Instrum. Meth. A 462, 152-155 (2001)

48. ALEPH collaboration, R. Barate et al., Study of charm production in Z decays. Eur. Phys. J. C 16, 597-611 (2000). https://doi.org/ 10.1007/s100520000421. arXiv:hep-ex/9909032
49. A. Meurer et al., Sympy: symbolic computing in python. Peer J. Comput. Sci. 3, e103 (2017). https://doi.org/10.7717/peerj-cs.103

50. A. Buckley et al., LHAPDF6: parton density access in the LHC precision era. Eur. Phys. J. C 75, 132 (2015). https://doi.org/10. 1140/epjc/s10052-015-3318-8. arXiv:1412.7420

51. G.A. Schuler, T. Sjöstrand, Parton distributions of the virtual photon. Phys. Lett. B 376, 193-200 (1996). https://doi.org/10.1016/ 0370-2693(96)00265-1. [arXiv:hep-ph/9601282

52. R. Frederix, S. Frixione, Merging meets matching in MC@NLO. JHEP 12, 061 (2012). https://doi.org/10.1007/JHEP12(2012)061. arXiv: 1209.6215

53. M.L. Mangano, M. Moretti, F. Piccinini, R. Pittau, A.D. Polosa, ALPGEN, a generator for hard multiparton processes in hadronic collisions. JHEP 0307, 001 (2003). [arXiv:hep-ph/0206293

54. M. Mangano, Merging multijet matrix elements and shower evolution in hadronic collisions. http://mlm.web.cern.ch/mlm/talks/ lund-alpgen.pdf (2004)

55. R. Frederix, S. Frixione, A. Papaefstathiou, S. Prestel, P. Torrielli, A study of multi-jet production in association with an electroweak vector boson. arXiv:1511.00847

56. L.A. Harland-Lang, A.D. Martin, P. Motylinski, R.S. Thorne, Parton distributions in the LHC era: MMHT 2014 PDFs. Eur. Phys. J. C 75, 204 (2015). https://doi.org/10.1140/epjc/s10052-015-3397-6. arXiv: 1412.3989

57. S. Dulat et al., The CT14 global analysis of quantum chromodynamics. arXiv: 1506.07443

58. P. Gras, S. Hoeche, D. Kar, A. Larkoski, L. Lönnblad, S. Plätzer et al., Systematics of quark/gluon tagging. arXiv:1704.03878

59. S. Catani, B.R. Webber, G. Marchesini, QCD coherent branching and semiinclusive processes at large $\mathrm{x}$. Nucl. Phys. B 349, 635-654 (1991). https://doi.org/10.1016/0550-3213(91)90390-J

60. J. Bellm, L. Gellersen, High dimensional parameter tuning for event generators. arXiv: 1908.10811

61. A. Denner, S. Dittmaier, L. Hofer, Collier: a fortran-based complex one-loop library in extended regularizations. Comput. Phys. Commun. 212, 220-238 (2017). https://doi.org/10.1016/j.cpc.2016.10. 013. [arXiv:1604.06792

62. A. Buckley et al., Rivet user manual. Comput. Phys. Commun. 184, 2803-2819 (2013). https://doi.org/10.1016/j.cpc.2013. 05.021. [arXiv: 1003.0694

63. M. Cacciari, G.P. Salam, G. Soyez, FastJet User Manual. Eur. Phys. J. C 72, 1896 (2012). https://doi.org/10.1140/epjc/ s10052-012-1896-2. [arXiv:1111.6097

64. A. Buckley, H. Hoeth, H. Lacker, H. Schulz, J.E. von Seggern, Systematic event generator tuning for the LHC. Eur. Phys. J. C 65, 331-357 (2010). https://doi.org/10.1140/epjc/ s10052-009-1196-7. [arXiv:0907.2973

65. ATLAS collaboration, M. Aaboud et al., Measurements of the production cross section of a $Z$ boson in association with jets in pp collisions at $\sqrt{s}=13 \mathrm{TeV}$ with the ATLAS detector. Eur. Phys. J. C 77, 361 (2017). https://doi.org/10.1140/epjc/s10052-017-4900-z. arXiv: 1702.05725 